\title{
Correction to: On the deviation of the lunar center of mass to the East. Two possible mechanisms based on evolution of the orbit and rounding off the shape of the Moon
}

\author{
B.P. Kondratyev ${ }^{1,2,3}$
}

Published online: 26 February 2019

(c) Springer Nature B.V. 2019

\section{Correction to: Astrophys Space Sci (2018) 363:186 https://doi.org/10.1007/s10509-018-3405-z}

After publication of the original article, the author made a series of checks on the main results. In general, the results of calculations were confirmed. Nevertheless, in numerical calculations in the second of the mechanisms presented there, an error was made (the deflection angle $\alpha$ was taken with the opposite sign), which eventually led to an incorrect result for $x=\frac{R_{\oplus \mathbb{I}}}{R_{\oplus}}$. After correction, the formula (48) will have the form

$\varepsilon(\alpha, E)=1-C \cdot \exp \left\{\left[\frac{\sin \alpha}{\cos (2 E-\alpha)}\right]^{\frac{1}{\cos 2 E}}\right\}$.

After amendment in the sign of the angle $\alpha$, Eq. (57) gives the value of $x=\frac{R_{\oplus \mathbb{B}}}{R_{\oplus}} \approx 3.233$ (instead of the previous $x \approx$ 1.43). The solution to Eq. (57) is shown in Fig. 1.

According to the corrected result, the Moon was formed in the annular zone at a distance of 3 to 4 average radii of the modern Earth. This result seems to be consistent with the modern idea that the Moon was formed as a result of a giant impact in the immediate vicinity of Proto-Earth.

The online version of the original article can be found under https://doi.org/10.1007/s10509-018-3405-z.

$\triangle$ B.P. Kondratyev

work@boris-kondratyev.ru

1 Sternberg Astronomical Institute, M.V. Lomonosov Moscow State University, 13 Universitetskij Prospect, Moscow, 119992, Russia

2 Faculty of Physics of the M.V. Lomonosov Moscow State University, 119991, Moscow, Russia

3 Central Astronomical Observatory at Pulkovo, Pulkovskoye chaussee 65, Saint-Petersburg, 196140, Russia

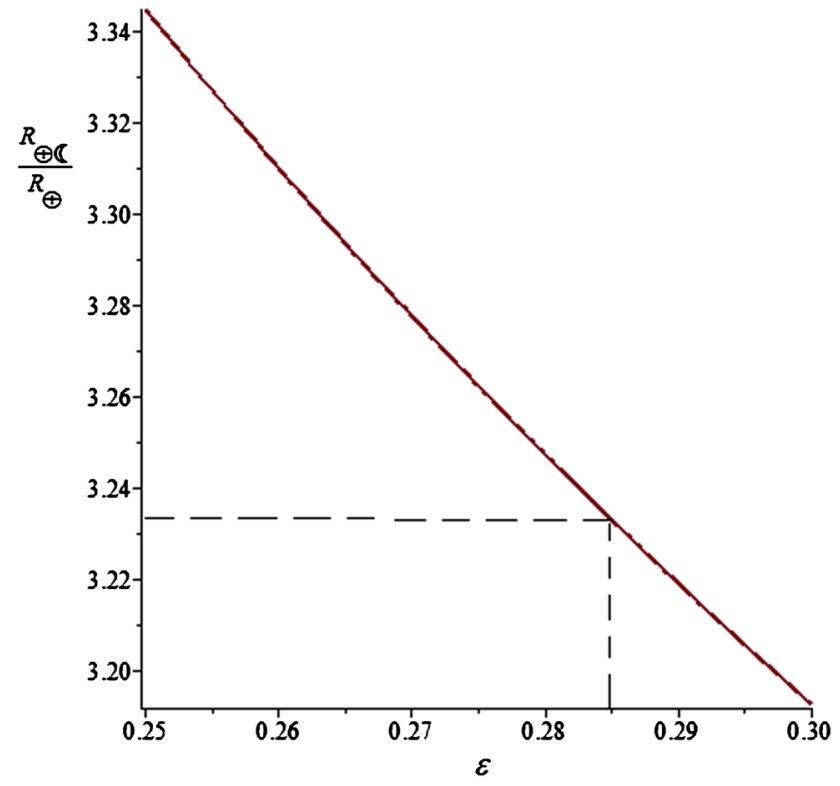

Fig. 1 The dependence of the normalized to $R_{\oplus}$ distance $R_{\oplus \bigotimes}$ from the Earth, on which the Moon could have been formed in the early era of the elongation $\varepsilon$ of its figure. The strokes show the results of calculations

Publisher's Note Springer Nature remains neutral with regard to jurisdictional claims in published maps and institutional affiliations. 